\title{
Development on ethanol production from xylose by recombinant Saccharomyces cerevisiae
}

\author{
Jin-Ying Yang, Jian-Ren Lu*, Hong-Yue Dang, Yan Li, Bao-Sheng Ge
}

Center for Bioengineering and Biotechnology of China University of Petroleum, Qingdao, China

Received 26 August 2009; revised 28 September 2009; accepted 30 September 2009.

\begin{abstract}
Xylose is the second major fermentable sugar present in lignocellulosic hydrolysates, so its fermentation is essential for the economic conversion of lignocellulose to ethanol. However, the traditional ethanol production strain Saccharomyces cerevisiae does not naturally use $x y-$ lose as a substrate. A number of different approaches have been used to engineer yeasts to reconstruct the gene background of $S$. cerevisiae in recent years. The recombinant strains showed better xylose fermentation quality by comparison with the natural strains. This review examines the research on $S$. cerevisiae strains that have been genetically modified or adapted to ferment xylose to ethanol from three aspects including construction of xylose transportation, xylose-metabolic pathway and inhibitor tolerance improvement of $S$. cerevisiae.
\end{abstract}

Keywords: Sacchromyces cerevisiae; Xylose; Ethanol; Metabolic Engineering

\section{INTRODUCTION}

Rising concerns over the cost of petroleum and the prospect of global warming are driving the development of technologies for the production of alternative fuels such as ethanol [1]. Cellulosic biomass is an attractive feedstock for fuel ethanol production since it is readily available, e.g., as a waste from the pulp and paper or agricultural industries, and also due to the fact that it is renewable with cycles many orders of magnitude shorter compared with those of fossil fuels. Hydrolysates of cellulosic biomass will contain mixtures of sugars, including glucose, galactose, mannose, xylose and arabinose, and other constituents in variable proportions depending on the source [2]. Successful industrial production of ethanol from lignocellulosic hydrolysate depends on the quantitative conversion of carbon present in the biomass.
It is well known that one of the most effective ethanol-producing organisms for hexose sugars is $\mathrm{Sac}$ charomyces cerevisiae, which shows high ethanol productivity, high tolerance to ethanol, and tolerance to inhibitory compounds present in the hydrolysate of lignocellulosic biomass [3-5]. However, S. cerevisiae does not naturally use xylose as a substrate. Only a few yeasts such as Pichia stipitis [6] and Pachysolen tannophilus [7] are able to ferment xylose. Genetic engineering can be used to enable $S$. cerevisiae to transport and ferment xylose including modeling, mutation, deletion and so on.

The pentose phosphate pathway (PPP) [8] is a process that serves to generate NADPH for reductive biosynthesis reactions within cells and the synthesis of pentose (5-carbon) sugars for the synthesis of the nucleotides and nucleic acids. There are two distinct phases in the pathway. The oxidative phase converts the hexose, D-glucose $6 \mathrm{P}$, into the pentose, D-ribulose 5P, plus $\mathrm{CO}_{2}$ and NADPH. The non-oxidative phase converts D-ribulose $5 \mathrm{P}$ into D-ribose 5P, D-xylulose 5P, D- sedoheptulose 7P, D-erythrose 4P, D-fructose 6P and D- glyceraldehyde 3P. $\mathrm{D}$-Xylose and L-arabinose enter the PPP through D-xylulose (Figure 1)

In bacteria, xylose is directly isomerized to xylulose by xylose isomerase (XI) before entering pentose phosphate pathway. In xylose-fermenting yeasts, xylose is first reduced to xylitol by xylose reductase (XR) and then oxidized to xylulose by xylitol dehydrogenase (XDH) [9]. Xylulokinase (XK) phosphorylates xylulose to xylulose 5-phosphate, which is then metabolized through the PPP and glycolysis (Figure 2). S. cerevisiae is not able to metabolize xylose due to the lack of XR and $\mathrm{XDH}$ activity, but it can utilize the isomeric form xylulose.

\section{ENGINEERING YEASTS FOR XYLOSE METABOLISM}

\subsection{Xylose Uptake}

Xylose is not readily fermentable in wild-type strains of $S$. cerevisiae. To circumvent this problem, different 


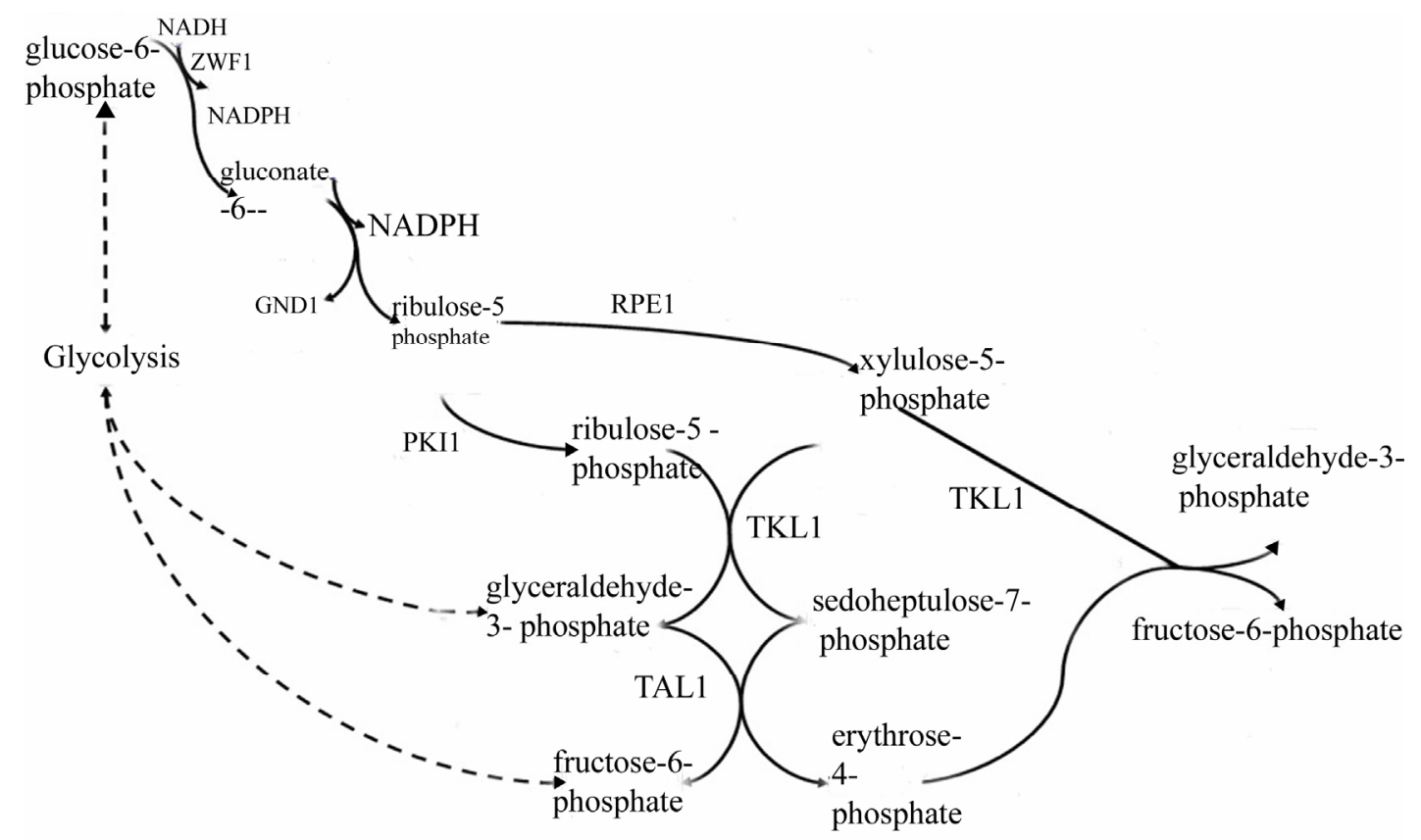

Engineering pentose metabolism in yeasts. The pentose phosphate pathway (PPP) in yeasts contains the oxidative phase, which consists of glucose 6-phosphate dehydrogenase (ZWF1) and 6-phosphogluconate dehydrogenase (GND1), and the non-oxidative phase, which is carried out by D-ribulose-5-phosphate 3-epimerase (RPE1), ribose-5-phosphate ketol-isomerase (RKI1), transketolase (TKL1) and transaldolase (TAL1).

Figure 1. The pentose phosphate pathway.

metabolic engineering strategies have been applied to enable xylose metabolism, and pentose-fermenting strains of $S$. cerevisiae have been created principally by engineering the pathways for converting xylose to xylulose-5-phosphate $[10,11]$. However, fermentation of xylose still remains significantly less efficient than that of glucose by these strains. The uptake of xylose into the cell is one of the reasons.

The $S$. ceresiave genome contains 20 genes that encode for hexose transporters but does not contain genes for xylose-specific transport system like natural xylose-utilizing yeasts [12]. Uptake of xylose by $S$. cerevisiae has been proposed to be mediated more or less unspecifically by its hexose-transport system. This is composed of a large family of 18 related transporter proteins called Hxts and additional sugar transporters with broader substrate specificity $[13,14]$.

Hamacher et al. [12] found that after deletion of all of the 18 hexose-transporter genes, the ability of $S$. cerevisiae cells to take up and to grow on xylose was lost. Re-introduction and constitutive expression of individual HXT genes in strain TMB3201 revealed that at 2\% xylose concentrations, high- (Hxt7 and Gal2) and intermediate-affinity (Hxt4 and Hxt5) glucose transporters are required for xylose uptake.

Several studies have indicated that in S. cerevisiae glucose and xylose appear to share the same transport facilities and competitively inhibit their mutual transport
$[15,16]$. Competition with glucose restricts xylose assimilation, so heterologous expression of a specific $x y-$ lose transporter could be very useful.

Researchers have tried to identify genes target for improved xylose assimilation. Two genes (GXF1 and GSX1) encoding xylose/glucose transporters from Candida in-

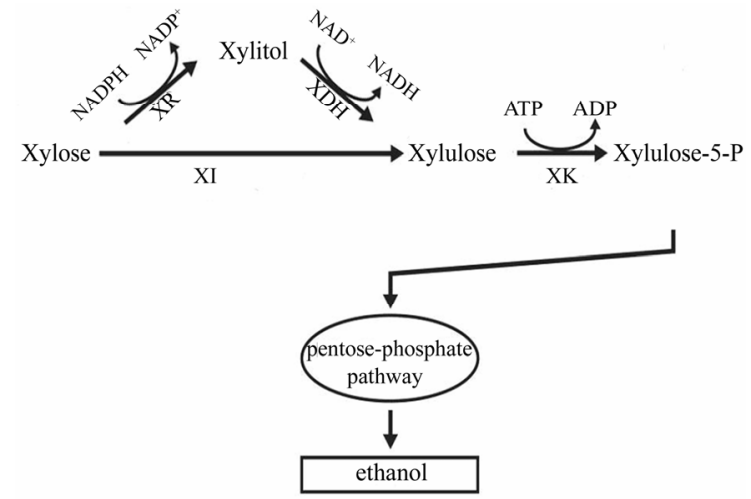

XR: Xylose reductase; XDH : xylitol dehydrogenase; XK : Xylulokinase

Figure 2. The metabolism of xylose in bacteria.

termedia were isolated by Leandro et al. [17], and expressed in S. cerevisiae. Gsxl is the first yeast xylose/glucose- $\mathrm{H}^{+}$symporter to be characterized in Arabidopsis thaliana at the molecular level. Except GSX1, xylose transporters from Arabidopsis thaliana 
(At5g59250) and Escherichia coli (xylE) were also expressed in $S$. ceresiave TMB3120 and failed to support vigorous growth of the recipient $S$. cerevisiae strain on xylose. Even though, the results warrant further investigations for the development of efficient bioethanol production processes from lignocellulosic materials.

The presence of three sugar transporters in P. stipitis, Sut1, Sut2 and Sut 3 has been reported. Although all three transporters have a higher affinity for glucose than for xylose, the Sut1 transporter has a higher Vmax for xylose uptake compared to other two Sut transporters and for hexose transporters [18,19]. Satoshi Katahira [20] et al. introduced SUT1 into a xylose-assimilating $S$. cerevisiae strain that expresses xylose reductase, xylosedehydrogenase and xylulokinase. The results showed that expression of Sut1 in xylose-assimilating $S$. cerevisiae increased both xylose uptake ability and ethanol productivity during xylose fermentation. Also, the enhancement of xylose uptake enables to accelerate the ethanol productivity during xylose/glucose co-fermentation. However, there are researchers with different opinions. Gárdonyi et al. [21,22] concluded that xylose transport in $S$. cerevisiae strains has low control over the rate of xylose utilization, unless the xylose pathway is significantly improved. In-depth study of the transporters' mechanism along with new modeling should continue to drive this field forward.

\subsection{Construction of Recomnant S. Cerevisiae Strains with Xylose-Fermenting Ability}

\subsubsection{Recombinant $S$. cerevisiae Expressing $X R$, $\mathrm{XDH}$, and XK}

Researchers have engaged in the development of engineered yeast strains capable of xylose fermentation by introducing XR and XDH into $S$. cerevisiae. Both of these enzymes have been isolated and characterized due to the central role they play in xylose metabolism.

The purified monomeric XR was NADPH-dependent with an apparent MW of $37 \mathrm{kDa}$, which was firstly purified and characterized by Kuhn et al. [23] XDHs also have been purified and characterized from various $x y-$ lose-fermenting yeasts.

Kötter et al. [24] first reported the construction of a $S$. cerevisiae strain expressing the $\mathrm{XR}$ - and $\mathrm{XDH}$-encoding genes XYL1 and XYL2 derived from the xylose-utilizing yeast $P$. stipitis. Walfridsson et al. [25] also genetically engineered $S$. cerevisiae to utilize xylose by introducing the XYL1 and XYL2 genes on either multicopy plasmids or by integrating them into the chromosome.

Although these strains can ferment xylose to ethanol, the excretion of xylitol occurs unless a co-metabolizable carbon source such as glucose is added. One of the most important reason is intercellular redox imbalance due to a different coenzyme specificity of xylose reductase (with $\mathrm{NADPH}^{+}$) and $\mathrm{XDH}\left(\right.$ with $\mathrm{NAD}^{+}$) [26]. Proteinengineering of $\mathrm{NADPH}^{+}$-preferring $\mathrm{XR}$ and/or $\mathrm{NAD}^{+}$dependent $\mathrm{XDH}$ is an alternative approach to solve the problem.

Anderlund [27] constructed four chimeric genes encoding fusion proteins of $X Y L 1$ and $X Y L 2$ with different orders of the enzymes and different linker lengths. These genes were expressed in S. cerevisiae. The fusion proteins exhibited both XR and XDH activity when XYL1 was fused downstream of XYL2. The results showed that the xylitol yield was lower in these strains than in strains expressing only native $\mathrm{XR}$ and $\mathrm{XDH}$ monomers, 0.55 and 0.62 , respectively, and the ethanol yield was higher.

By analyzing the amino acid of coenzyme-binding domain of XDH, Watanabe [28] modified XDH from $P$. stipitis by three- and four-site direct mutagenesis. The triple mutant (D207A/I208R/F209S) and quadruple mutant (D207A/I208R/F209S/N211R) showed more than 4500 -fold higher values in $\mathrm{kcat} / \mathrm{Km}$ with $\mathrm{NADP}^{+}$than the wild-type enzyme, reaching values comparable with $\mathrm{kcat} / \mathrm{Km}$ with $\mathrm{NAD}^{+}$of the wild-type enzyme.

In recent years, the research group introduced these mutated PsXDHs with the PsXR WT to $S$. cerevisiae and estimated effect(s) of the functional modification(s) of PsXDH on fermentation of xylose to ethanol in recombinant $S$. cerevisiae [29]. The results showed that recombinant yeast strains gave the highest ethanol production and the lowest xylitol excretion.

Zeng et al. [30] altered the coenzyme specificity of $P$. stipitis XR via rational design based on the 3D structure. Lys 21 , the only one amino acid that has hydrogen binding interaction with $\mathrm{NADP}^{+}$but not with $\mathrm{NAD}^{+}$in the binding pocket, were changed to Ala and Arg respectively. The results showed that the coenzyme dependence of K21A was completely reversed to $\mathrm{NADH}^{+}$.

\subsubsection{Recombinant S. cerevisiae Expressing Xylose Isomerase}

Xylose isomerase (XI), encoded by the xylA gene, catalyzes the isomerization of xylose to xylulose in bacteria and some fungi [31]. xylA has been cloned into $S$. cerevisiae from several bacteria. However the XI produced by the recombinant $S$. cerevisiae strains was inactive. Improper protein folding, postranslational modifications, inter- and intramolecular disulfide bridge formation, and the internal $\mathrm{pH}$ of yeast have been suggested as possible reasons [32].

In 1996, Walfridsson et al. [33] cloned the Thermus thermophilus xylA gene encoding xylose (glucose) isomerase and successfully expressed in $S$. cerevisiae under the control of the yeast $P G K 1$ promoter. The recombinant xylose isomerase showed the highest activity at $85^{\circ} \mathrm{C}$ with a specific activity of $1.0 \mathrm{U} / \mathrm{mg}$. It was the first successful attempt to express the procaryotic gene $x y l A$ for the enzyme XI in the eucaryote $S$. cerevisiae, 
which could be due to the relatedness between the two organisms. The recombinant strains could not covert xylose to ethanol efficiently because the temperature and $\mathrm{pH}$ optimum for the recombinant enzyme are high.

The XylA gene from the anaerobic fungus Piromyces sp. E2 (ATCC 76762) was functionally expressed in $S$. cerevisiae by Marko Kuyper et al. [34]. After prolonged cultivation on xylose, a mutant strain was obtained that grew aerobically and anaerobically on xylose. The anaerobic ethanol yield was $0.42 \mathrm{~g}$ ethanol $/ \mathrm{g}$. xylose and also by-product formation was comparable to that of glucose-grown anaerobic cultures.

In 2009, Brat et al. [35] cloned and successfully expressed a highly active new kind of xylose isomerase from the anaerobic bacterium Clostridium phytofermentans in S. cerevisiae. The recombinant yeast cells with heterologous expression got the ability to metabolize D-xylose and to use it as the sole carbon and energy source. The new enzyme has low sequence similarities to the XI Thermus thermophilus and Piromyces sp. E2, which were the only two xylose isomerases previously functionally expressed in $S$. cerevisiae. Importantly, the new enzyme is far less inhibited by xylitol, which accrues as a side-product during xylose fermentation. The findings provided an excellent starting point for further improvement of xylose fermentation in industrial yeast strains.

\section{OPTIMIZATION OF DOWNSTREAM METABOLIC PATHWAYS}

\subsection{Xylulokinase}

Although recombinant strains containing genes coding for XR and XDH from the xylose-utilizing yeast $P$. stipitis have been reported, such strains ferment xylose to ethanol poorly. One reason for this may be the low capacity of xylulokinase, the third enzyme in the xylose pathway [36,37].

Xylulokinase is an enzyme that catalyzes the chemical reaction: ATP + D-xylulose $\rightleftharpoons$ ADP + D-xylulose 5-phosphate. In 1989, Ho et al. [38] cloned the xylulokinase (xks1) gene from $S$. cerevisiae and firstly overexpressed in $S$. cerevisiae. Toivari et al. [39] also overexpressed the endogenous gene for xylulokinase (xks1) in $S$. cerevisiae along with the $P$. stipitis genes for XR and XDH. The metabolism of this recombinant yeast was further investigated in pure xylose bioreactor cultivation at various oxygen levels. The results clearly indicated that overexpression of $x k s 1$ significantly enhanced the specific rate of xylose utilization. In addition, the XK-overexpressing strain can more efficiently convert xylose to ethanol under all aeration conditions studied. These two studies represented an important step in efforts to improve xylose metabolism in $S$. cerevisiae, as their results strongly indicated that native XK activity was insufficient for xylose or xylulose fermentation, and overexpression was required to obtain high ethanol yields.

\subsection{Transketolase and Ransaldolase}

Transketolase and transaldolase catalyze transfer of 2-C and 3-C molecular fragments respectively, in each case from a ketose donor to an aldose acceptor. The two enzymes have been implicated as being rate-limiting for xylose and xylulose fermentation.

The TKL1 and TAL1 genes encoding transketolase and transaldolase were overexpressed individually and together in the $S$. cerevisiae strain containing XYL and XYL2. The strain overexpressing TAL1 showed considerably enhanced growth on xylose compared with a strain containing only XYL1 and XYL2. Overexpression of only TKL1 did not influence growth. The results indicated that the transaldolase level in S. cerevisiae is insufficient for the efficient utilization of pentose phosphate pathway metabolites [40]. Bao et al. [41] also found that the $S$. cerevisiae strain overexpressing the TAL1 and TKL1 showed considerably good growth on the xylose plate.

\section{IMPROVEMENT OF TOLERANCE TO INHIBITORS}

The growth of $S$. cerevisiae and ethanol production were limited by multiple inhibitors, including furan derivatives, 5-hydroxymethylfurfural (HMF), weak acids, and phenolic compounds produced during biomass-to-ethanol processing. The PPP is an important pathway incarbohydrate metabolism, and a lot of previous studies have shown a correlation between several PPP genes and specific stresses such as oxidative [42], sorbic acid [43], and osmotic [44].

To improve production of fuel ethanol from renewable raw materials, laccase from the white rot fungus Trametes versicolor was expressed under control of the $P G K 1$ promoter in $S$. cerevisiae to increase its resistance to phenolic inhibitors in lignocellulose hydrolysates [45]. To identify target genes involved in furfural tolerance, Gorsich [46] screened a $S$. cerevisiae gene disruption library for mutants with growth deficiencies in the presence of furfural. As a result, more than 62 genes were found to be associated with sensitivity to furfural. They also further showed that overexpression of ZWF1 in $S$. cerevisiae allowed growth at furfural concentrations that are normally toxic, which demonstrated a strong relationship between PPP genes and furfural tolerance.

Adapting strains is also an alternative to improve the performance of microorganisms. Liu [47] improved bio- 
transformation by newly developed strains adapted to tolerate the challenges of furfural and HMF in batch cultures compared with the parental strains. The results suggest a possible in situ detoxification of the inhibitors for bioethanol fermentation using improved yeast strains. Although they have not been tested against inhibitor complexes such as those in a biomass hydrolysate, the development and study of such strains provided necessary materials for further studies of the mechanisms of the stress tolerance at molecular and genomic levels.

\section{CONCLUSIONS}

The bioconversion of cellulose and hemicellulose to biofuels and chemicals is being actively researched with the aim of developing technically and economically viable processes. D-Xylose is the major product of the hydrolysis of hemicellulose and considerable research efforts has been focused on the development of xylosefermenting recombinant $S$. cerevisiae. Significant improvements in ethanol productivity from xylose have been achieved through metabolic engineering. However, there are still unidentified limiting steps in the xylose metabolism of metabolically engineered $S$. cerevisiae, such as lower ethanol yield, more byproducts, the suitability of these recombinant strains and so on. There are still many tasks that left in the xylose-metabolic engineering. So far the recombinant $S$. cerevisiae were constructed base on the laboratory strains, which are less complex in genetic background, growth characters, and physiological characters comparing with the industrial yeast strains. To get strains easy to be industrialized, more emphasis should be focused on the reconstruction of the wild type yeasts. Further improve the expression and stability of the heterogenous genes in yeasts can be expected for higher ethanol yield.

\section{REFERENCES}

[1] A. J. Ragauskas, C. K. Williams, B. H. Davison, et al. (2006) The path forward for biofuels and biomaterials. [J]. Science, 311, 484-489.

[2] A. Wiselogel, S. Tyson, and D. Johnsson, (1996) Biomass feedstock resources and composition. [M]. C. E.Wyman (Editor), Handbook on bioethanol: Production and utilization. Washington, Taylor and Francis, 105-118.

[3] L. Olsson and B. Hahn-Hägerdal, (1993) Fermentative performance of bacteria and yeast in lignocellulose hydrolysates. [J]. Process Biochemistry, 28(4), 249-257.

[4] L. Olsson and J. Nielsen, (2000) The role of metabolic engineering in the improvement of Saccharomyces cerevisiae: Utilization of industrial media. [J]. Enzyme Microbial Technology, 26(9-10), 785-792.

[5] B. Hahn-Hägerdal, C. F. Wahlbom, M. Gárdonyi, et al., (2001) Metabolic engineering of Saccharomyces cerevisiae for xylose utilization. Advances in Biochemistry
Engineering and Biotechnology, 73, 53-84.

[6] T. W. Jeffries, I. V. Grigoriev, J. Grimwood, et al., (2007) Genome sequence of the lignocellulose-bioconverting and xylose-fermenting yeast Pichia stipitis. [J]. Nature Biotechnology, 25, 319-326.

[7] P. J. Slininger, R. J. Bothast, J. E. Vancauwenberge, et al., (1982) Conversion of D-xylose to ethanol by the yeast Pachysolen tannophilus [J]. Biotechnology and Bioengineering, 24(2), 371-384.

[8] T. W. Jeffries, (2006) Engineering yeasts for xylose metabolism. [J]. Current Opinion in Biotechnology, 17, 320- 326.

[9] K. L. Träff, R. R. O. Cordero, W. H. van Zyl, et al., (2001) Deletion of the GRE3 aldose-reductase gene and its influence on xylose metabolism in recombinant strains of Saccharomyces cerevisiae expressing the xylA and Xks1 genes. [J], Applied and Environmental Microbiology, 67(12), 5668-5674.

[10] A. Eliasson, C. Christensson, C. F. Wahlbom, et al., (2000) Anaerobic xylose fermentation by Recombinant Saccharomyces cerevisiae Carrying XYL1, XYL2 and XKS1 in Mineral Medium Chemostat Cultures. [J]. Applied and Environmental Microbiology, 66(8), 3381-33-86.

[11] M. Kuyper, M. M. P. Hartog, M. J. oirkens, et al., (2005) Metabolic engineering of a xylose-isomerase-expressing Saccharomyces cerevisiae strain for rapid anaerobic xylose fermentation. [J]. FEMS Yeast Research, 5(4-5), 399-409.

[12] T. Hamacher, J. Becker, M. Gárdonyi, et al., (2002) Characterization of xylose-transporting properties of yeast hexose transporters and their influence on xylose utilization. [J]. Microbiology, 148, 2783-2788.

[13] E. Boles and C. P. Hollenberg, (1997) The molecular genetics of hexose transport in yeasts. [J]. FEMS Microbiology Reviews, 21(1), 85-111.

[14] R. Wieczorke, S. Krampe, T. Weierstall, et al., (1999) Concurrent knock-out of at least 20 transporter genes is required to block uptake of hexoses in Saccharomyces cerevisiae. [J]. FEBS Letter, 464, 123-128.

[15] A. Kotyk, (1968) Properties of the sugar carrier in baker's yeast. [J]. Folia Microbiologica, 13(3), 212-220.

[16] P. Kötter and M. Ciriacy, (1993) Xylose fermentation by Saccharomyces cerevisiae $[\mathrm{J}]$. Applied Microbiology and Biotechnology, 38(6), 776-783.

[17] L. M. José, P. Goncalves, and I. Spencer-Martins, (2006) Two glucose / xylose transporter genes from the yeast Candida intermedia: First molecular characterization of a yeast xylose $-\mathrm{H}^{+}$symporter. [J]. Biochemistry Journal, 395(3), 543-549.

[18] T. Weierstall, C. P. Hollenberg, and E. Boles, (1999) Cloning and characterization of three genes (SUT1-3) encoding glucose transporters of the yeast Pichia stipitis. [J]. Molecular Microbiology, 31(3), 871-883.

[19] T. Hamacher, J. Becker, M. Gárdonyi, et al., (2002) Characterization of the xylose-transporting properties of yeast hexose transporters and their influence on xylose utilization [J]. Microbiology, 148, 2783-2788.

[20] S. Katahira, M. Ito, H. Takema, et al., (2008) Improvement of ethanol productivity during xylose and glucose co-fermentation by xylose-assimilating $S$. cerevisiae via expression of glucose transporter Sut1 [J]. Enzyme and Microbial Technology, 43(2), 115-119. 
[21] M. Gárdonyi, M. Jeppsson, G. Lidén, et al., (2003) Control of xylose consumption by xylose transport in recombinant Saccharomyces cerevisiae. [J]. Biotechnology and Bioengineering, 82(7), 818-824.

[22] M. Gárdonyi, M. Österberg, C. Rodrigues, et al., (2003) High capacity xylose transport in Candida intermedia PYCC 4715. [J]. FEMS Yeast Research, 3, 45-52.

[23] A. Kuhn, C. van Zyl, A. van Tonder, et al., (1995) Purification and partial characterization of an aldo-keto reductase from Saccharomyces cerevisiae. [J]. Applied and Environmental Microbiology, 61(4), 1580- 1585.

[24] P. Kötter, R. Amore, C. P. Hollenberg, et al., (1990) Isolation and characterization of the Pichia stipitis xylitol dehydrogenase gene, XYL2, and construction of a xylose-utilizing Saccharomyces cerevisiae transformant. [J]. Current Genetics, 18(6), 493-500.

[25] M. Walfridsson, J. Hallborn, M. Penttila, et al., (1995) Xylose-metabolizing Saccharomyces cerevisiae strains overexpressing the TKL1 and TAL1 genes encoding the pentose phosphate pathway enzymes transketolase and transaldolase. [J]. Applied and Environmental Microbiology, 61(12), 4184-4190.

[26] S. Ostergaard, L. Olsson, and J. Nielsen, (2000) Metabolic Engineering of Saccharomyces cerevisiae. [J]. Microbiology and Molecular Biology Reviews, 64(1), 34- 50.

[27] M. Anderlund, P. Rådström, and B. H. Hägerdal, (2001) Expression of bifunctional enzymes with Xylose Reductase and Xylitol Dehydrogenase activity in Saccharomyces cerevisiae alters product formation during Xylose fermentation. [J]. Metabolic Engineering, 3(3), 226-235.

[28] S. Watanabe, T. Kodaki, and K. Makino, (2005) Complete reversal of coenzyme specificity of xylitol dehydrogenase and increase of thermostability by the introduction of structural zinc. [J]. The Journal of Biological Chemistry, 280(11), 10340-10349.

[29] S. Watanabe, A. A. Salehb, S. P. Pack, et al., (2007) Ethanol production from xylose by recombinant Saccharomyces cerevisiae expressing protein engineered $\mathrm{NADP}^{+}$-dependent xylitol dehydrogenase. [J]. Journal of Biotechnology, 130,316-319.

[30] Q. K. Zeng, H. L. Du, Z. C. Zhai, et al., (2008) Mutational research on the role of Lysine 21 in the Pichia stipitis Xylose Reductase. [J]. Chinese Journal of Biotechnology, 24(6), 1108-1111.

[31] T. W. Jeffries and Y. S. Jin, (2000) Ethanol and thermotolerance in the bioconversion of xylose by yeasts [J]. Advances in Applied Microbiology, 47, 221-268.

[32] R. Amore, M. Wilhelm, and C. P. Hollenberg, (1989) The fermentation of xylose-An analysis of the expression of Bacillus and Actinoplanes xylose isomerase gens in yeast. [J]. Applied Microbiology and Biotechnology, 30(4), 351-357.

[33] M. Walfridsson, X. Bao, M. Anderlund, et al., (1996) Ethanolic fermentation of xylose with Saccharomyces cerevisiae harboring the Thermus thermophilus xylA gene, which expresses an active xylose (glucose) isomerase. [J]. Applied and Environmental Microbiology, 62(12), 4648-4651.

[34] M. Kuyper, A. A. Winkler, J. P. van Dijken, et al., (2001) Minimal metabolic engineering of Saccharomyces cerevisiae for efficient anaerobic xylose fermentation: a proof of principle. [J]. FEMS Yeast Research, 4(6), 655-664.
[35] D. Brat, E. Boles, and B. Wiedemann, (2009) Functional expression of a bacterial xylose isomerase in Saccharomyces cerevisiae. [J]. Applied and Environmental Microbiology, 75(8), 2304-2311.

[36] Y. S. Jin and T. W. Jeffries, (2003) Changing flux of xylose metabolites by altering expression of xylose reductase and xylitol dehydrogenase in recombinant Saccharomyces cerevisiae. [J]. Applied Biochemistry and Biotechnology, 106(1-3), 277-285.

[37] X. D. Xue and N. W. Y. Ho, (1990) Xylulokinase activity in various yeasts including Saccharomyces cerevisiae containing the cloned xylulokinase gene. [J]. Applied Biochemistry and Biotechnology, 24-25(1), 193-199.

[38] N. W. Y. Ho and S. F. Chang, (1989) Cloning of yeast xylulokinase gene by complementation in Escherichia coli and yeast mutations. [J]. Enzyme Microbial Technology, 11, 417-421.

[39] M. H. Toivari, A. Aristidou, L. Ruohonen, et al., (2001) Conversion of Xyl ose to ethanol by recombinant Saccharomyces cerevisiae: importance of Xylulokinase (XKS1) and oxygen availability. [J]. Metabolic Engineering, 3(3), 236-249.

[40] M. Walfridsson, J. Hallborn, M. Penttilä, et al., (1995) Xylose-metabolizing Saccharomyces cerevisiae strains overexpressing the TKL1 and TAL1 genes encoding the pentose phosphate pathway enzymes transketolase and transaldolase. [J]. Applied and Environmental Microbiology, 61(12), 4184-4190.

[41] X. Bao, D. Gao, Y. Qu, et al., (1997) Effect on product formation in recombinant Saccharomyces cerevisiae strains expressing different levels of xylose metabolic genes. [J]. Chinese Journal of Biotechnology, 13(4), 225- 231.

[42] H. Juhnke, B. Krems, P. Kotter, et al., (1996) Mutants that show increased sensitivity to hydrogen peroxide reveal an important role for the pentose phosphate pathway in protection of yeast against oxidative stress. [J]. Molecular \& General Genetics, 252(4), 456-464.

[43] M. Mollapour, D. Fong, K. Balakrishnan, et al., (2004) Screening the yeast deletant mutant collection for hypersensitivity and hyperresistance to sorbate, a weak organic acid food preservative. [J]. Yeast, 21(11), 927-946.

[44] B. Krems, C. Charizanis, and K. D. Entian, (1995) Mutants of Saccharomyces cerevisiae sensitive to oxidative and osmotic stress. [J]. Current Genetics, 27(5), 427434.

[45] S. Larsson, P. Cassland, and L. J. Jonsson, (2001) Development of a Saccharomyces cerevisiae strain with enhanced resistance to phenolic fermentation inhibitors in lignocellulose hydrolysates by heterologous expression of laccase. [J]. Aplied and Environmental Microbiology, 67(3), 1163-1170.

[46] S. W. Gorsich, B. S. Dien, N. N. Nichols, et al., (2006) Tolerance to furfural-induced stress is associated with pentose phosphate pathway genes $Z W F 1, G N D 1, R P E 1$, and TKL1 in Saccharomyces cerevisiae. [J]. Applied Microbiology and Biotechnology, 71(3), 339-349.

[47] Z. L. Liu, P. J. Slininger, and S. W. Gorsich, (2005) Enhanced biotransformation of furfural and hydroxymethylfurfural by newly developed ethanologenic yeast strains. [J]. Applied Biochemistry and Biotechnology, 121(1-3), 451-460. 